\title{
Position statement on opportunistic genomic screening from the Association of Genetic Nurses and Counsellors (UK and Ireland)
}

\author{
Anna Middleton ${ }^{\star, 1}$, Chris Patch ${ }^{2}$, Jennifer Wiggins ${ }^{3}$, Kathy Barnes ${ }^{4}$, \\ Gill Crawford $^{5}$, Caroline Benjamin ${ }^{6}$, Anita Bruce ${ }^{7}$, on behalf of the \\ Association of Genetic Nurses and Counsellors in the United Kingdom \\ and Ireland
}

The American College of Medical Genetics and Genomics released recommendations for reporting incidental findings (IFs) in clinical exome and genome sequencing. These suggest 'opportunistic genomic screening' should be available to both adults and children each time a sequence is done and would be undertaken without seeking preferences from the patient first. Should opportunistic genomic screening be implemented in the United Kingdom, the Association of Genetic Nurses and Counsellors (AGNC), which represents British and Irish genetic counsellors and nurses, feels strongly that the following must be considered (see article for complete list): (1) Following appropriate genetic counselling, patients should be allowed to consent to or opt out of opportunistic genomic screening. (2) If true IFs are discovered the AGNC are guided by the report from the Joint Committee on Medical Genetics about the sharing of genetic testing results. (3) Children should not be routinely tested for adult-onset conditions. (4) The formation of a list of variants should involve a representative from the AGNC as well as a patient support group. (5) The variants should be for serious or life-threatening conditions for which there are treatments or preventative strategies available. (6) There needs to be robust evidence that the benefits of opportunistic screening outweigh the potential harms. (7) The clinical validity and utility of variants should be known. (8) There must be a quality assurance framework that operates to International standards for laboratory testing. (9) Psychosocial research is urgently needed in this area to understand the impact on patients.

European Journal of Human Genetics (2014) 22, 955-956; doi:10.1038/ejhg.2013.301; published online 8 January 2014

Keywords: AGNC; opportunistic screening; incidental finding; position statement; genomics n March 2013 the American College of
Medical Genetics and Genomics (ACMG)
released recommendations for reporting
incidental findings (IFs) in clinical exome
and genome sequencing; these were then published in July 2013. ${ }^{1}$ These advocated that laboratories performing clinical sequencing should deliberately search for and report on a pre-determined list of cancer and cardiac IFs (including conditions

\footnotetext{
${ }^{1}$ Human Genetics, Wellcome Trust Sanger Institute, Cambridge, UK; ${ }^{2}$ Department of Clinical Genetics, Guys Hospital, London, UK; ${ }^{3}$ Cancer Genetics Department, The Royal Marsden NHS Foundation Trust, Surrey, UK; ${ }^{4}$ Teesside Genetics Unit, James Cook University Hospital, Teeside, UK; ${ }^{5}$ CELS (Clinical Ethics and Law at Southampton)Wessex Clinical Genetics Service, Faculty of Medicine, Southampton General Hospital, Southampton, UK; ${ }^{6}$ Transforming Health Evidence Group, School of Health, University of Central Lancashire, Preston, UK; ${ }^{7}$ North East Thames Regional Genetics Service, Great Ormond Street Hospital, London, UK

${ }^{*}$ Correspondence: $\operatorname{Dr}$ A Middleton, Wellcome Trust Sanger Institute, Cambridge CB10 1SA, UK. Tel: +44 1223 492391; Fax: +44 1223 494919; E-mail: Am33@sanger.ac.uk

Received 7 November 2013; revised 25 November 2013; accepted 29 November 2013; published online 8 January 2014
}

with onset in either childhood or adulthood) in addition to the results directly relevant to the initial reason for referral. According to the ACMG, such 'opportunistic genomic screening' should be available to both adults and children and would be undertaken without seeking preferences from the patient first.

This represents a significant change from the current approach in health care where, in general, a patient presents to a clinician with a specific medical question that needs answering. In answering that question other findings may emerge; if so the clinician will likely interpret and manage these. Unless the nature of the consultation is specifically to screen for a collection of diseases, in most clinical settings the clinician would not routinely search for additional findings, unrelated to the original medical question. In most publicly funded health-care systems, such as the National Health Service (NHS) in the United Kingdom, screening programmes in healthy individuals are the subject of scrutiny, quality assurance and include a broad assessment of costs and benefits.

Over the last 5 years, there has been much support in the ethics, policy and medical literature for feeding back genomic IFs in both clinical and research settings; the premise being that in addition to explore a genetic diagnosis pertinent to the patient's phenotype, sequencing technologies serendipitously afford an opportunity to seek out and identify other predispositions, for example to life-threatening preventable conditions. In clinical practice at present a patient undergoes genetic testing because of an existing phenotype in themselves or their family; opportunistic genomic screening has the potential to identify predispositions to serious disease in an otherwise healthy person who may have no existing phenotype in themselves or their family. Thus implementing opportunistic genomic screening and managing patients accordingly would be an extension to the current practice.

The main objective of the Association of Genetic Nurses and Counsellors (AGNC) is to advance and enhance the standard of patient care for those seeking genetic counselling, including diagnosis of, or screening for, genetic disease. The AGNC recognises that whole-genome/-exome sequencing technologies will become a part of routine clinical practice across many areas of medicine. As both targeted and non-targeted genetic tests will increasingly be used in the clinic, many more patients will have to deal with unexpected results and will require expert clinical input. Genetic counsellors are specifically 
trained to support patients and their families as they process and use genetic information; they are voluntarily registered in the United Kingdom by the Genetic Counsellor Registration Board to enhance patient safety and accountability. Genetic counsellors are also experts in helping patients and their families communicate about genetics and deal with the inherent uncertainties surrounding this type of information.

As genomic medicine develops, patients are more likely to be given risk information about multiple conditions. The genetic counsellor is well placed to help them and their wider family navigate their way through this information. Genetic counsellors are also familiar with working long term with families and are able to follow up individuals with genetic results as they reach different stages in their lives (eg, when making reproductive choices). In addition to working directly with patients, the genetic counsellor also has an important part to play in educating other health professionals who will be implementing genomic medicine in other areas of health care.

The following recommendations were created by a Working Group convened by the AGNC and chaired by the first author. The Working Group consisted of members of the AGNC, who volunteered their participation. A call for representation from the membership was made by the AGNC Chair at the annual AGNC conference in 2013. The Working Group met on one occasion for a day to discuss the ACMG recommendations and explore how these related to practice in the United Kingdom and Ireland. At the end of this day, consensus was reached on an appropriate list of recommendations deemed important to genetic counselling practice, should opportunistic screening be introduced into the United Kingdom. These were collated by the first author and sent to the AGNC Committee for further discussion. The AGNC Committee ratified the document after making minor amendments. These were then published on the AGNC website (www.agnc.org.uk).

\section{RECOMMENDATIONS}

Should opportunistic genomic screening be implemented in practice in the United Kingdom, the AGNC feels strongly that the following must be considered:

- Individual autonomy is at the heart of genetic counselling practice. Following appropriate genetic counselling, patients should be allowed to consent to or opt out of receiving IFs offered as part of opportunistic genomic screening. The laboratory will then test for and /or analyse what the patient has consented to; this helps to avoid the situation of the laboratory (and clinician) potentially knowing results that the patient has specifically asked not to receive.

- If true IFs are discovered throughout the course of any testing, that are indeed a surprise to a patient and clinician, the AGNC are guided by the report from the Joint Committee on Medical Genetics about the sharing of genetic testing results with the individual and family. ${ }^{2}$

- In accordance with numerous International guidance on the genetic testing of children, children should not routinely be tested for adult-onset conditions. The AGNC adheres to guidance written by our constituent group, the British Society for Genetic Medicine (formally the British Society of Human Genetics) on the genetic testing of children. ${ }^{3}$

- If there were a pre-determined list of variants that could be reported on in an opportunistic genomic screen in the UK, the formation of this list should be consensus-driven and include a representative from the AGNC as well as a patient support group. It should also be guided by the principles that currently underpin National and International guidelines on disease screening.

- The variants should be for serious or lifethreatening conditions for which there are treatments, interventions or preventative strategies available.
- There needs to be robust evidence that the benefits of opportunistic screening outweigh the potential harms in terms of over-diagnosis, unnecessary treatment and the possibility of psychological harm.

- The clinical validity and utility of variants should be known and we acknowledge that this is difficult in the absence of extensive population studies.

- Testing should only be offered within a quality assurance framework that operates to International standards for laboratory testing.

- Opportunistic genomic screening will increase the need for disease screening services and it may not be possible to immediately meet this need in a publicly funded service such as the NHS. Thus, there needs to be capacity in the NHS to meet screening demand and the associated downstream costs.

- There is an urgent need for empirical research into the psychosocial and organisational aspects of care with regards to integrating opportunistic genomic screening into practice.

\section{CONFLICT OF INTEREST}

The authors declare no conflict of interest.

1 Green RC, Berg JS, Grody WW et al: 'ACMG recommendations for reporting of incidental findings in clinical exome and genome sequencing.' Genet Med 2013; 15 565-574.

2 Royal College of Physicians, Royal College of Pathologists, British Society for Human Genetics 2011

'Consent and confidentiality in clinical genetic practice guidance on genetic testing and sharing genetic information.' Report of the Joint Committee on Medical Genetics 2nd edn. Available from http://www.bsgm. org.uk/media/678746/consent_and_confidentiality_ 2011.pdf.

3 British Society of Human Genetics 2010 'Report on the genetic testing of children.' Retrieved 25/11/13, available from http://www.bsgm.org.uk/media/678741/ gtoc_booklet_final_new.pdf. 